\title{
Study on Transformer Fault Diagnosis Based on Dynamic Fault Tree
}

\author{
Fei Peng ${ }^{1,2}$, Lin Cheng ${ }^{1,2}$, Kaikai Gu², Zhenbo Du ${ }^{2}$, Jiang Guo ${ }^{3}$ \\ ${ }^{1}$ School of Electrical and Electronic Engineering, Huazhong University of Science and Technology, Wuhan, China \\ ${ }^{2}$ Wuhan Nari Group Corporation of State Grid Electric Power Research Institute, Wuhan, Hubei, China \\ ${ }^{3}$ School of Power and Mechanical Engineering, Wuhan University, Wuhan, Hubei, China
}

Email address:

759733212@qq.com (Fei Peng), chenglin@nari-ge.com (Lin Cheng),gkkyf@126.com (Kaikai Gu), 52781821@qq.com (Zhenbo Du), guo.river@163.com (Jiang Guo)

\section{To cite this article:}

Fei Peng, Lin Cheng, Kaikai Gu, Zhenbo Du, Jiang Guo. Study on Transformer Fault Diagnosis Based on Dynamic Fault Tree. Journal of Electrical and Electronic Engineering. Vol. 3, No. 5, 2015, pp. 133-138. doi: 10.11648/j.jeee.20150305.16

\begin{abstract}
In this paper, according to theoretical diagnosis of fault tree, the author builds a diagnosis model based on dynamic fault tree and illustrates the model's construction method and diagnosis logic in detail. According to case analysis, compared with conventional fault tree diagnosis, the above-mentioned method is advanced in fault-tolerant ability. Plus, the diagnosis results record some intermediate processes of the diagnosis, with relevant information being returned to the researchers as ideas facilitating further analysis in the event of incomplete information.
\end{abstract}

Keywords: Transformer, Dynamic Fault Tree, Fault-Tolerant Ability, Fault Diagnosis

\section{Introduction}

Since the operation state of the transformer has a direct bearing on the security and stability of the whole power grid, it is necessary to prevent and minimize the occurrence of transformer faults and accidents to the largest degree. In recent years, having been used for transformer fault diagnosis, with certain achievements being achieved, various intelligent techniques ${ }^{[1-5]}$ have provided bases for transformer fault diagnosis. Among many types of intelligent techniques, fuzzy set and fault tree analysis are the two categories applied and researched most. But mostly in transformer fault diagnosis, simplex intelligent techniques are used for this purpose, the final judgments of which lack guiding significance with respect to the formulation of maintenance strategies. In actual operating process, transformer faults are usually characterized by complexity, uncertainty and concurrency of multiple faults, in which, the use of simplex intelligent fault diagnosis will cause such problems as low accuracy, poor reasoning, and so on, difficult to obtain satisfactory diagnostic effect, especially for fault tree analysis and fuzzy mathematics theory. In fault diagnosis process, fault tree is poor in interpretation, hard to accurately and quantitatively describe the occurrence possibility of fault tree nodes, with a transition zone existing between "healthy" state and "faulty" state, and in the case of incomplete information, fuzzy set theory is not possible be determined.

Aiming at using the appropriate combination of fuzzy set and fault tree, study on transformer fault diagnosis based on the two concepts mutually makes up their deficiencies with their advantages, in order to overcome the respective disadvantages of fuzzy set or fault tree and improve the accuracy and efficiency of transformer fault diagnosis.

\section{Construction of Transformer Fault Tree}

Study on fault diagnosis based on the fault tree principle has made a large number of research results, with many successful cases occurring in industrial field. However, through years of application researches, following defects or deficiencies are also exposed gradually: 1) Precise fault positioning is not possible; 2) Fault tree node lacks relevant information required in fault detection; 3) It is hard to accurately and quantitatively describe the possibility of the occurrence of fault tree nodes.

\subsection{Dynamic Fault Tree Nodes}

In this paper, to overcome deficiencies of fault tree applied 
in transformer fault diagnosis, the author proposes dynamic fault tree nodes, as shown in Figure 1, based on a comprehensive reference to conventional fault tree theory and the actual situation of transformer fault diagnosis.

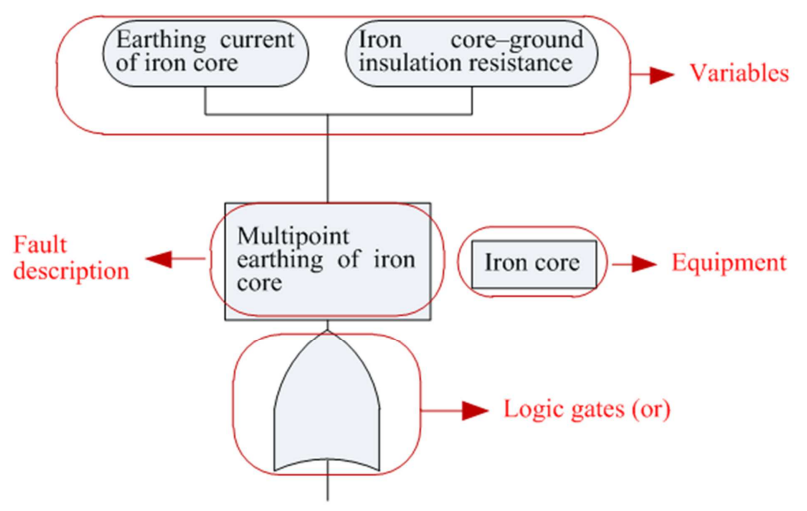

Fig. 1. A dynamic fault tree node with variables.

Compared with conventional fault tree nodes, dynamic fault tree nodes have introduced 2 parts, namely, "variables" and "equipment".

1) Variables refer to the relevant information needed in fault diagnosis, and these characteristics that have shown are the "variables" of this system. Along with the occurrence of each fault, there will be some phenomenon, such as "temperature rise", "overcurrent", and "partial discharge volume exceeding the standard". The introduction of variables is to connect the system in series successfully. When received by the system, original-state data of the transformer (inspection, online monitoring, test data, and so on) will be first processed through a series of procedures (eliminating algorithm, regression algorithm, trend analysis, and so on) to turn from original data into state information, which, then, will be processed and transferred into variables of fault tree for serving the diagnosis of the whole fault tree. Secondly, another advantage of introducing variables is that: in the event that the conclusion obtained from fault tree diagnosis is indeterminate, the corresponding state quantity can be traced according to the fault tree node variables obtained through diagnosis, with further analysis being performed based on respective test recommendations of the state quantity. Variables are also a key to make fuzzy input of fault tree possible, which will be discussed hereinafter.

2) Equipment refers to components or sub-components of the fault tree node which are connected to the transformer. Eventually, fault diagnosis is a troubleshooting service, and it will achieve precise fault positioning by connecting the fault tree node to the equipment. Troubleshooting personnel can locate the specific parts requiring repair or replacement according to this information.

Table 1. List of variables for dynamic fault tree node.

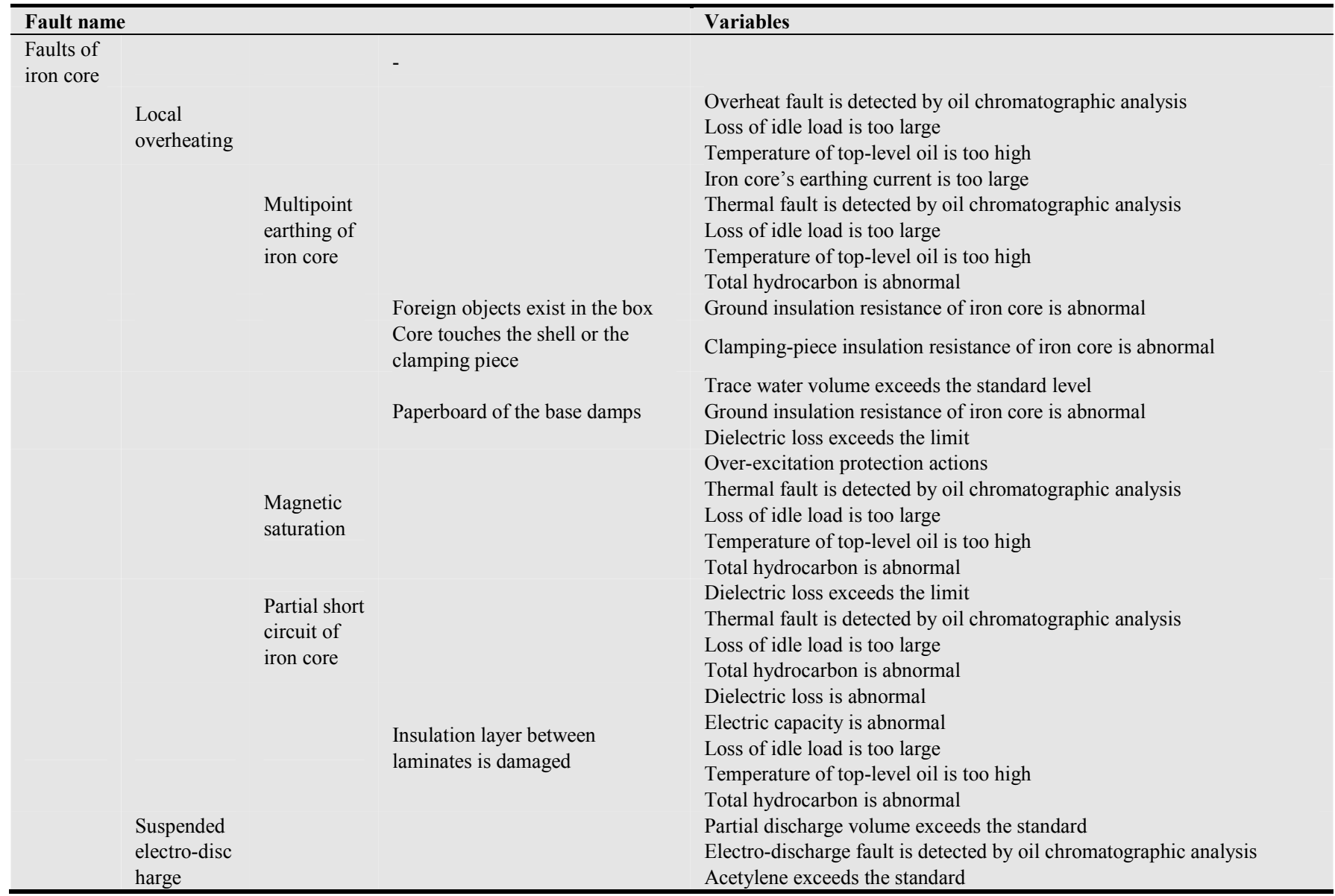




\subsection{Construction of Transformer Fault Tree}

Systematic research analysis is the key to build a transformer fault tree. Small components or faults of high frequency should not be ignored simply, because some small faults are probably the root causes of serious system faults. Since transformer is of a complex structure with many sub-components, "modular decomposition" should be used. This method is to divide transformer into 8 subsystems first, namely, iron core, winding, insulation oil, casing, cooling system, tap switch, and non-electricity protection, and analyze each sub-system and build subtrees according to principles of building fault tree, and then combine these subtrees together. After constructing the fault tree completely, determine for each fault tree node the relevant state quantity, namely, "variables", which is as shown in Table 1.

\section{Fault Diagnosis Based on Dynamic Fault Tree}

\subsection{Establishment of State Set}

Carry out state evaluation for indicators, which can be divided into 4 levels, with their respective possible words being "healthy", "attentive", "abnormal", and "faulty". Given that the state set is:

$$
V=\left\{V_{1}, V_{2}, V_{3}, V_{4}\right\}
$$

In this formula: $V_{i}(i=1,2,3,4)$ refers to State Level i. To make full use of information provided by the state evaluation, set the corresponding point of the given state levels $1-4$ as 1.00 $0.75,0.5$, and 0.25 .

\subsection{Determination of Membership Degree}

Since membership function is the basis of applying fuzzy set, whether the membership function is constructed correctly [6] is one of the key factors to make good use of fuzzy set. Most of the variables of transformer fault tree nodes are measurable values, namely quantitative variables, which can be divided into 3 categories: the smaller the better, the nearer to the middle the better, and the bigger the better. For variables of the smaller the better, its membership function adopts formula (2). For variables of the bigger the better, its membership function usually adopts formula (3).

$$
A(x)=\left\{\begin{array}{cc}
1 & x \leq a \\
f(x) & x>a
\end{array}\right.
$$

In this formula: $f(x)$ is a nonincreasing function, with $0 \leq$ $f(x) \leq 1$.

$$
A(x)=\left\{\begin{array}{cc}
1 & x \leq a \\
g(x) & x>a
\end{array}\right.
$$

In this formula: $g(x)$ is a nondecreasing function, with $0 \leq$ $g(x) \leq 1$.

For variables of the nearer to the middle the better, its membership function can be demonstrated by the distribution of middle-type fuzzy functions.

\subsection{Fuzzy State Matrix of Dynamic Fault Tree Nodes}

State of dynamic fault tree nodes is directly related to variables, and Dynamic fault tree node F contains $\mathrm{k}$ (a number) variables. Thus, $\mathrm{F}$ can be divided into the following $\mathrm{k}$ sets of divisors and factors:

$$
F=\left\{F_{1}, F_{2}, \cdots, F_{k}\right\}
$$

Among them, the simplex-factor state matrix of subset $i$ is as follows:

$$
F_{i}=\left[F_{i 1}, F_{i 2}, F_{i 3}, F_{i 4}\right]
$$

In this formula: $F_{i j}(\mathrm{i}=1,2, \ldots, \mathrm{k} ; \mathrm{j}=1,2,3,4)$ refers to the corresponding membership of Node F's Variable i to State Level $V_{j}$.

According to the above analysis, the fuzzy state matrix of Node F is obtained as follows:

$$
R_{k \times 4}=\left[\begin{array}{cccc}
F_{11} & F_{12} & F_{13} & F_{14} \\
F_{21} & F_{22} & F_{23} & F_{24} \\
\ldots & \ldots & \ldots & \ldots \\
F_{k 1} & F_{k 2} & F_{k 3} & F_{k 4}
\end{array}\right]
$$

When the fuzzy state matrix of dynamic fault tree nodes needs to be obtained based on the simplex-factor state matrix of variables, the weight set of variables is required to be known. Fuzzy mathematical operation is performed based on the fuzzy matrix of variables and its weight set, which will lead the fuzzy state matrix of dynamic fault tree nodes being obtained.

\subsection{Determination of Variables' Weights by Analytic Hierarchy Process}

To get variables' weights of dynamic fault tree nodes, it is necessary to establish a judgment matrix. For example, to get the corresponding weight of such variables as that "overheat fault is detected by oil chromatographic analysis", "loss of idle load is too large", and "temperature of top-level oil is too high" to the dynamic fault tree node "local overheating", it is necessary to compare the significance of these three kinds of variables for "local overheating" respectively, with the compared results being based to form a $3 \times 3$ judgment matrix ${ }^{[6-7]}$. By finding the corresponding eigenvector to the largest eigenvalue of the matrix, it is available to get the corresponding weights of the three variables to the dynamic fault tree node "local overheating".

In formula (4), the general procedures of getting the 
corresponding weight of Variable $F_{\mathrm{i}}(i=1,2, \cdots, k)$ to dynamic fault tree node $\mathrm{F}$ are as follows:

1) Invite an expert to compare the significance of $F_{i}$ for $\mathrm{F}$ respectively, the basis of which is shown in Table 2[14];

Table 2. List of scale.

\begin{tabular}{ll}
\hline Scale & Degree Description \\
\hline 1 & Equally important \\
3 & Somewhat more important \\
5 & Obviously more important \\
\hline
\end{tabular}

2) Make judgment according to the rules given by Table 2, and establish a judgment matrix $H_{k \times k}$ based on the respectively compared results, among which, Factor $a_{\mathrm{ij}}(i, j=1,2, \cdots, k)$ refers to the result of comparing variable $F_{\mathrm{i}}$ with variable $F_{j}$, with $a_{\mathrm{ii}}=1, a_{\mathrm{ij}}=1 / a_{j i}$. For example, when $a_{12}=1$, it means $F_{1}$ and $F_{2}$ are equally important.

3) In the consistency test for the judgment matrix, the information of analytic hierarchy process comes from the judgment of experts on the relative importance between each two factors on one level. After quantization by appropriate scale, the information will form a judgment matrix. When any factor in Matrix $H_{k \times k}$ meets the condition of $a_{\mathrm{ij}}=a_{\mathrm{il}} a_{\mathrm{lj}}(i, j, l=1,2, \cdots, k)$, it means that the matrix has certain consistency. As for a consistent matrix, it is available to obtain the weight of each indicator by getting the corresponding characteristic quantity to its maximum feature root. For those inconsistent cases, it is necessary to adjust the judgment matrix so as to achieve consistency, the specific adjustment method of which is shown in Reference [8] and Reference [9].

4) Establish weight set for indicators. After getting the consistent Judgment Matrix $\mathrm{H}$, take its corresponding eigenvectors $\left[w_{1}, w_{2}, \cdots, w_{k}\right]$ to its Maximum Feature Root $\lambda$ as weight coefficients, with $\sum_{i=1}^{k} w_{\mathrm{i}}=1$, where $w_{\mathrm{i}}$ refers to the weight of variable $i$ with respect to the influence on dynamic fault tree node. If $\mathrm{H}$ is not a consistent one, the corresponding normalized eigenvectors to its maximum feature root should be taken as Weight Set $w$, with the following formula coming into being:

$$
H w^{T}=\lambda w^{T}
$$

\subsection{State Evaluation on Dynamic Fault Tree Nodes}

After obtaining the simplex-factor state matrix of each variable and determining the weight vectors of each layer's indicators, it is available to adopt fuzzy mathematical operation to get the fuzzy state set of the dynamic fault tree node. Max-min compositional operation is performed:

$$
F=w_{1 \times k} \circ R_{k \times 4}
$$

In this formula: $F$ is the fuzzy matrix on the judgment level of the dynamic fault tree node; $w_{1 \times k}$ is the corresponding membership degree of the variable to dynamic fault tree node $F$; and $R_{k \times 4}$ is the fuzzy state matrix of the variable.

In $F$, the computational formula of each Factor $F_{i}$ is as follows:

$$
F_{j}=\mathrm{i}_{\mathrm{i}=1}^{\mathrm{k}}\left(\mathrm{w}_{\mathrm{i}} \Lambda r_{\mathrm{ij}}\right) \mathrm{i}=1,2, . ., \mathrm{k} ; \mathrm{j}=1,2,3,4
$$

In this formula: $w_{i}$ and $r_{i j}$ are factors in Matrixes $w_{1 \times k}$ and $R_{k \times 4}$, with $\mathrm{V}$ and $\Lambda$ representing supremum and infimum respectively.

According to the rules of highest membership degree, the corresponding state level to the largest element in the matrix is the state evaluation level of the dynamic fault tree node. Put the value of the state level in the following form of computational vectors:

$$
Q=\left[\begin{array}{llll}
1 & 0.75 & 0.5 & 0.25
\end{array}\right]
$$

Normalize the Fuzzy Judgement Matrix $F$ obtained based on the formula, and get Matrix $F^{\prime}$. Multiply $F^{\prime}$ with $Q$, which will get the evaluation score $\mathrm{S}$ of this dynamic fault tree node:

$$
S=F^{\prime} Q^{T}
$$

\subsection{Fault Reasoning Strategies}

The specific processes and strategies of transformer fault diagnosis based on compact fusion of fuzzy set and fault tree are as follows:

1) Build transformer fault tree, set membership function for each variable, and then establish weight relationship for variables of dynamic fault tree nodes according to the actual conditions.

2) As for fuzzy state matrix of state quantity, input the test value for the state quantity, and get its fuzzy state matrix based on its membership function.

3) As for the state judgment for dynamic fault tree node, based on the corresponding Weight Set $w$ of the state quantity to the dynamic fault tree node, the state score of the dynamic fault tree node is obtained according to Formula (8) and Formula (9), which, thus, will lead the state of the dynamic fault tree node being obtained.

4) As for fault tree diagnosis, a variable will be used in more than one dynamic fault tree node. As shown in Table 1, the variable "temperature of top oil layer" is associated to such dynamic fault tree nodes as "local overheating", "multipoint earthing of iron core", "magnetic saturation", and "insulation layer between laminates being damaged". It is available to get dynamic fault tree node states caused by a set of variables through Step 2) and Step 3), with several diagnosis branches being formed on the fault tree.

5) As for fault positioning, in fault tree diagnosis, if all branches can be positioned to the root causes, then they should be traced back to the corresponding root causes. However, for transformer, such a complex system, which is often 
impossible to realize precise fault positioning through one diagnosis mode, needs to be supported by other detection and experiment methods, with branches of root causes not positioned being returned to unidentified variables of the next layer of the dynamic fault tree node. Then, put values of these variables and initial state quantities into Step 2), and repeat Step 3), Step 4), and Step 5) until all branches are positioned to root causes.

\section{Diagnosis Examples and Result Analysis}

A real diagnosis example is given below. The state information of a transformer is that oil chromatographic analysis shows overheating fault, excess loss of idle load, and extremely high temperature of the top oil level. According to Table 1, the current fault is local overheating, which is that the dynamic fault tree node "local overheating" is lightened. Now, it is required to identify the root cause of the fault. The specific steps are as follows:

1) Focus on the variables on the next level of the dynamic fault tree node "local overheating", and test its value by being supported by other means. The processes of calculating the state matrix for the iron core earthing current are given below, and the type of the iron core earthing current is the indicator of the smaller the better. Theoretically, completely healthy iron core earthing current tends to 0 , and for transformers of small capacity, generally it is regarded as a healthy one when the iron core earthing current is lower than $90 \mathrm{~mA}$. Roughly, it can be defined that the iron core earthing currents of 90,100 and 110 correspond to 1-4 grade respectively. So that, it is available to get the Membership Function $A_{i}(\mathrm{x})$ of iron core earthing current belonging to the Fuzzy Set $V_{i}(i=1,2,3,4)$ as follows:

$$
\begin{aligned}
& A_{1}(\mathrm{x})=\left\{\begin{array}{cc}
1 & 0 \leq x \leq 90 \\
\frac{100-x}{100-90} & 90 \leq x \leq 100 \\
0 & x \geq 100
\end{array}\right. \\
& A_{2}(\mathrm{x})=\left\{\begin{array}{cc}
\frac{x}{90} & 0 \leq x \leq 90 \\
1 & 90 \leq x \leq 100 \\
\frac{110-x}{110-100} & 100 \leq x \leq 110 \\
0 & x \geq 110
\end{array}\right. \\
& A_{3}(\mathrm{x})=\left\{\begin{array}{cc}
0 & 0 \leq x \leq 90 \\
\frac{100-x}{100-90} & 90 \leq x \leq 100 \\
1 \quad 100 & \leq x \leq 110 \\
\frac{110}{x} & x \geq 110
\end{array}\right.
\end{aligned}
$$

$$
A_{4}(\mathrm{x})=\left\{\begin{array}{cl}
0 & 0 \leq x \leq 100 \\
\frac{110-x}{110-100} & 100 \leq x \leq 110 \\
1 & x \geq 110
\end{array}\right.
$$

The measured value of iron core earthing current is $45 \mathrm{~mA}$, after which is put into these 4 functions, the state matrix of iron core earthing current will be $[1,0.5,0,0]$.

2) Measure the score of the lower-level node ("multipoint earthing of iron core", "magnetic saturation", "partial short circuit of iron core" and "poor heat dissipation"). Take the calculation of the score of "multipoint earthing of iron core" as an example. The state matrixes of iron core earthing current, oil chromatographic analysis, idle-load loss, top-level oil temperature, and total hydrocarbon are $[1,0.5,0,0],[0,0,0.5,1]$, $[0,0,0.5,1],[0,0,0.25,1]$, and $[0,0.5,1,0.5]$ respectively, with the Fuzzy Matrix $R_{5 \times 4}$ based on Formula (6) being as follows:

$$
R_{5 \times 4}=\left[\begin{array}{cccc}
1 & 0.5 & 0 & 0 \\
0 & 0 & 0.5 & 1 \\
0 & 0 & 0.5 & 1 \\
0 & 0 & 0.25 & 1 \\
0 & 0.5 & 1 & 0.5
\end{array}\right]
$$

The corresponding Weight Judgment Matrix $H_{5 \times 5}$ of five variables to the dynamic fault tree node "multipoint earthing of iron core" is as follows:

$$
H_{5 \times 5}=\left[\begin{array}{ccccc}
1 & 2 & 2 & 2 & 2 \\
1 / 2 & 1 & 1 & 1 & 1 \\
1 / 2 & 1 & 1 & 1 & 1 \\
1 / 2 & 1 & 1 & 1 & 1 \\
1 / 2 & 1 & 1 & 1 & 1
\end{array}\right]
$$

It is shown that $H_{5 \times 5}$ has consistency, and Weight Set $w$ is obtained based on Formula (7):

$$
w=\left[\begin{array}{lllll}
0.36 & 0.16 & 0.16 & 0.16 & 0.16
\end{array}\right]
$$

The Fuzzy Judgment Matrix $F$ of dynamic fault tree node "multipoint earthing of iron core":

$$
\begin{aligned}
F & =\left[\begin{array}{lllll}
0.36 & 0.16 & 0.16 & 0.16 & 0.16
\end{array}\right] \circ\left[\begin{array}{cccc}
1 & 0.5 & 0 & 0 \\
0 & 0 & 0.5 & 1 \\
0 & 0 & 0.5 & 1 \\
0 & 0 & 0.25 & 1 \\
0 & 0.5 & 1 & 0.5
\end{array}\right] \\
& =\left[\begin{array}{llll}
0.36 & 0.16 & 0.16 & 0.16
\end{array}\right]
\end{aligned}
$$

After normalization, $F^{\prime}=\left[\begin{array}{llll}0.43 & 0.19 & 0.19 & 0.19\end{array}\right]$

According to Formula (10), score $\mathrm{S}$ is:

$$
\begin{aligned}
& S=\left[\begin{array}{llll}
0.43 & 0.19 & 0.19 & 0.19
\end{array}\right]\left[\begin{array}{llll}
1 & 0.75 & 0.5 & 0.25
\end{array}\right]^{T} \\
& =0.715
\end{aligned}
$$


It is shown that the state of dynamic fault tree node "multipoint earthing of iron core" is attentive. Similarly, the states of dynamic fault tree nodes "magnetic saturation", "partial short circuit of iron core", and "poor heat dissipation" are "attentive", "faulty", and "faulty" respectively.

3) As for unidentified variables the returned diagnosis results of which are root causes "poor heat dissipation" and "partial short circuit of iron core", since the partial circuit of the iron core is the fault node in the middle, it requires further observation on the state of its next-level node, which means to repeat Step (1) and Step (2) until the root causes are identified.

Discussions on Results: The interpretation ability of conventional fault tree diagnosis is low, with the results being normal or abnormal only. Meanwhile, the diagnostic capacity is low when information is incomplete. According to the above calculation example, conventional fault tree diagnosis shows the result of "poor heat dissipation". Compared with conventional fault diagnosis, the transformer fault diagnosis based on compact fusion of fuzzy set and fault tree is stronger in fault-tolerant ability, with the diagnosis results recording some intermediate processes of the diagnosis, which will return the relevant variables to the researchers as ideas for their further analysis in the case of incomplete information.

\section{Conclusion}

In this paper, based on respective advantages of fuzzy set and fault tree, the author presents the transformer fault diagnosis based on compact fusion of fuzzy set and fault tree, with case analysis demonstrating the accuracy being enhanced by this method for the transformer diagnosis.

1) Equipment and variables are introduced to the conventional dynamic fault tree node, with the precise positioning of fault diagnosis results and the combination of equipment state information and fault tree being achieved.

2) Construction method of the improved fault tree is described, with the flexibility and scalability of constructing fault tree being improved.

3) The compact fusion of the fuzzy set theory and dynamic fault tree nodes is realized, which brings fuzzy input to dynamic fault tree nodes, together with more states (healthy, attentive, abnormal, faulty), more consistent with the actual process.

4) Diagnosis capacity of the fault tree in the case of incomplete information gets improved. When information does not support the fault tree to locate the root cause, the variable will be returned to the lower-level dynamic fault tree node directly for providing ideas for researchers' further analysis.

As for the application of fuzzy set and fault in transformer diagnosis, the research methods and conclusions of this paper play important role both in terms of reference and practical engineering.

\section{References}

[1] FU Qiang, CHEN Tefang, ZHU Jiaojiao. Transformer Fault Diag-nosis Using Self-adaptive RBF Neural Network Algorithm[J]. High Voltage Engineering, 2012, 38(6): $1368-1375$.

[2] CHEN Xiaoqing, LIU Juemin, HUANG Yingwei, FU Bo. Transformer Fault Diagnosis Using Improved Artificial Fish Swarm with Rough Set Algorithm[J]. High Voltage Engineering, 2012, 38(6): 1403-1408.

[3] BAI Cuifen, GAO Wensheng, JIN Lei, YU Wenxuan, ZHU Wenjun. Integrated Diagnosis of Transformer Faults Based on Three-layer Bayesian Network[J]. High Voltage Engineering, 2013, 39(2): 330-335.

[4] Wang Jianyuan, Ji Yanchao. Appliacation of fuzzy petri nets knowledge representation in electric power transformer fault diag-nosis[J]. Proceedings of the CSEE, 2003, 23(3): 121-125.

[5] ZHAO Wenqing, ZHANG Shenglong, NIU Dongxiao. Transformer fault diagnosis based on multi-Agent[J]. Electric Power Automation Equipment, 2011, 31(3): 23-26.

[6] Lei Yaguo. Research on hybrid intelligent technique and its applications in fault diagnosis[D]. Xi'an, China: Xi' an Jiaotong University, 2007.

[7] Xie Jijian, Liu Chengping. Fuzzy mathematics method and its application[M]. Wuhan, China: Huazhong University of Science and Technology Press, 2000.

[8] Lan Jibin, Xu Yang, Huo Liangan, Liu Jiazhong. Research on the Priorities of Fuzzy Analytical Hierarchy Process [J]. Systems Engineering - Theory \& Practice, 2006, 26(9):107-112.

[9] SATTY T.L. The analytic hierarchy process.New York, NY, USA: McGraw-Hill Inc, 1980.

[10] Wang Yingluo. Systems engineering[M]. Beijing, China: Machinery Industry Press, 2001. 